\title{
Experimental measurements of the RF sheath thickness with a cylindrical Langmuir probe
}

\author{
E. Faudot, ${ }^{1} \mathrm{~J}_{\text {. Ledig, }}{ }^{1} \mathrm{~J}$. Moritz$^{1}{ }^{1} \mathrm{~S}$. Heuraux $^{1}{ }^{1} \mathrm{~N}$. Lemoine, ${ }^{1}$ and S. Devaux ${ }^{1,2}$ \\ ${ }^{1)}$ Institut Jean Lamour, Campus Artem, 2 allee André Guinier, 54011 Nancy, \\ France \\ ${ }^{2)}$ CRYOSCAN, Campus Artem, 2 allee André Guinier, 54011 Nancy, France
}

The small layer oscillating in front of a radio-frequency (rf) biased electrode in an asymmetric $\mathrm{rf}$ plasma discharge without magnetic field is diagnosed using a rf compensated cylindrical probe. Thanks to this probe $(0.15 \mathrm{~mm}$ in diameter $)$, the floating potential is measured in this area. Radiofrequency plasmas and sheath properties are then derived from the I-V characteristics measured by the probe at different rf power levels in both capacitive and direct couplings. In direct coupling the plasma biasing is, as expected, nearly equal to the applied rf potential except at high power levels for which the current collected by the electrode saturates and the sheath potential gap is reversed. In capacitive coupling, the self biasing of the electrode is strongly negative due to the matching box used. From the difference between the plasma potential and the floating potential we found sheath thickness of about $3 \lambda_{D e}$. Within the rf power scan performed, the sheath thicknesses deduced from potential and density profiles are 3 times higher than the Child Langmuir law both in direct and capacitive coupling in a low collisional Helium plasma.

\section{INTRODUCTION}

Diagnosing radio frequency (rf) plasmas is a rather complicated task even without magnetic field and the study of RF sheaths by probes is still a challenge even if $\mathrm{rf}$ discharges have been deeply studied ${ }^{1}$. In the literature, one can find out the main characteristics of an unmagnetized rf plasma discharge, collisional or not, symmetric or asymmetric, in direct or capacitive coupling. Lieberman ${ }^{2}$ and Chabert ${ }^{3}$ described the $^{2}$ main characteristics of rf discharges, especially rf sheath which are characterized by a typical thickness, capacitance and resistance. One of the first to investigate rf sheath properties is Godiak ${ }^{4}$ whose numerous works have modeled and measured the sheath thickness and capacitance.

The main consequence of the oscillating sheath close to a rf electrode is the self biasing either of the electrode itself in the case of a capacitive coupling, or of the plasma itself in direct coupling. By allowing dc current to flow through the electrode (direct coupling) or not leads to a very different biasing which in turn modifies the sputtering. This biasing comes from the rectification of the rf potential by the sheath, due to the non linearity of its capacitance and conductance ${ }^{5}$. This non linear response is simply given by the Boltzmann density distribution for electron in a collisionless sheath.

The sheath is a thin space charged layer that can be defined by its typical thickness and voltage drop between its edge and the wall, which can be biased (dc or rf sheath) or not (floating sheath). The sheath edge, or its thickness, is defined through the Bohm criterion which is fulfilled when the ion velocity is larger than their acoustic speed at the sheath edge. This criterion has been refined by ${ }^{6}$ but no simple analytical solution really exists for the sheath thickness, except for the well known Child Langmuir law, which provides the sheath width as a function of the sheath potential (much higher than the floating potential) with no collision ${ }^{3}$. For the case of a floating sheath, the reader should refer to ${ }^{7}$ in which the thickness is calculated from a fluid model and leads to an approximate sheath width of $5 \lambda_{D e}$ where $\lambda_{D e}$ is the Debye length.

In the context of a rf discharge, many models have been developed: collisionless ${ }^{8}$ and collisional sheath ${ }^{9}$ as well as an improved fluid model with arbitrary collision parameters ${ }^{10}$. These models provide the main properties of the sheath: conductance, capacitance, thickness, and the average potential drop as a function of the rf potential. The latest numerical calculations seem to show that the rf sheath thickness is much larger than the one given by the Child Langmuir law ${ }^{11}$. Other models are more suitable to calculate the time averaged rf sheath potential in an asymmetric capacitive rf discharges ${ }^{12,13}$ or in the case of the instantaneous rectified potential ${ }^{14-16}$. The model presented in ${ }^{14}$ is also valid with direct current (blocking capacitor short circuited). In the case of an asymmetric rf discharge with direct coupling, an exact solution of the instantaneous $r$ sheath potential as a function of the areas and capacitances of both sheaths is given in ${ }^{17}$, in which an asymmetric double probe model with capacitive rf currents is used.

Other attempts to measure the sheath thickness have already been made but they were using different methods in different plasmas. A review of the main ways to diagnose the sheath (dc and rf) are given in reference ${ }^{18}$. The first observation of the sheath width was made by Langmuir in Mercury discharges. One of the main contributors in the field is Godyak ${ }^{10}$, who provided a very complete model for the sheath 
capacitance and thickness, corroborated by measurements. These measurements, also performed in a Mercury vapor plasma in a symmetric rf discharge, consisted in measuring the sheath capacitance to deduce the sheath thickness. Another method uses emissive probes to measure directly the plasma potential in dc sheath ${ }^{19}$ and rf sheath ${ }^{20}$ in a multidipole plasma and at low frequencies (lower than $1 \mathrm{MHz}$ ). A third method is based on Laser Detachment Signal and is compared to probe measurements and Child Langmuir law ${ }^{21}$. These measurements were performed in a weakly magnetized plasma around a cylindrical biased probe. A fourth method consists in measuring the electric field in a rf sheath thanks to an electron beam probe in a rf parallel plate capacitive discharge in an Argon plasma ${ }^{22}$. Recently, ${ }^{23}$ retrieves the sheath width from the collected ion current on a cylindrical probe surface.

Langmuir probe theory is still mainly based on the first paper from Langmuir ${ }^{24}$ who first described the currents collected by different kind of biased probes (plane, cylindrical and spherical) in a potential well: the Orbital Motion Limit (OML) theory. Other theories have then been elaborated by many authors leading to the Allen-Boyd-Reynolds (ABR ${ }^{25}$ theory. This theory is dedicated to spherical probes and extended to cylindrical probes ${ }^{26}$. One can also cite the Bernstein-Rabinowitz ${ }^{27}$ theory for monoenergetic ions, refined by Laframboise ${ }^{28}$, but it is much more complex to apply. Another method just takes into account the sheath expansion theory ${ }^{29}$ to calculate the true collecting area of the probe. Our rf compensated ${ }^{30}$ cylindrical probe was manufactured by the Hiden Company and automatized by the Cryoscan company. The rf compensation has been changed to compensate frequencies from 25 to $60 \mathrm{MHz}$. Nevertheless the choice of chokes to cut off rf currents must be done carefully to be really efficient ${ }^{31}$.

Our experimental setup, plasma chamber, rf electrode and probe are described in the first section. The second section is dedicated to probe measurements and the methods used to deduce the plasma parameters from the current-voltage (I-V) characteristics. The third section deals with experimental results and presents potential profiles inside and outside the rf sheath. The last section is dedicated to the comparison of measured and theoretical sheath thicknesses with different techniques to find out the sheath edge.

\section{EXPERIMENTAL SETUP}

All measurements have been completed in the linear experimental device Aline ${ }^{32}$ depicted in figure 1. This is a cylindrical chamber of $1 \mathrm{~m}$ long and $30 \mathrm{~cm}$ in diameter surrounded by 6 axial coils able to produce a maximum $0.12 \mathrm{~T}$ magnetic field (in the frame of the present paper, the plasma is not magnetized). It is also equipped with a $600 \mathrm{~W}$ broadband (from $10 \mathrm{kHz}$ to $250 \mathrm{MHz}$ ) $\mathrm{RF}$ amplifier driven by a frequency synthetiser. Such a RF amplifier is usually used in RF antenna applications for telecommunications. Here, it is connected to the RF electrode (a stainless steal disc of $8 \mathrm{~cm}$ in diameter and $1 \mathrm{~cm}$ thick, as shown in figure 3) via a RF coupler able to measure both forward and reflected power and via a matching box able to match at frequencies from 10 to $40 \mathrm{MHz}$, depending on the plasma discharge parameters. The amplifier is also protected against reflected power up to $400 \mathrm{~W}$. In addition, a 3 axis manipulator developed by Cryoscan holds the Langmuir probe. It can move by $+/-5 \mathrm{~cm}$ along $\mathrm{x}$ and y axis (radial), and by $50 \mathrm{~cm}$ along $\mathrm{z}$ axis (axial). This allows to build potential profiles in the plasma or even 3D maps [? (devauxPSI) The probe acquisition system, the manipulator, the frequency synthetiser and the oscilloscope are all driven by a single software in order to control all the instruments at the same time to automatize the acquisition. As a matter of fact the manual operation would require too much time according to the many parameters to control: power and frequency (synthetiser), I-V characteristics (probe), probe position (manipulator), and even magnetic field strength in case of magnetized plasmas. The gas pressure is 3.4 Pa for all discharges presented here.

\section{A. Capacitive and direct coupling}

Two choices are possible to perform RF plasma discharges. The first consists in a direct coupling (see figure 2), where the amplifier is directly connected to the electrode via the coupler. The second one is a capacitive coupling, where a matching box is inserted between the coupler and the electrode to avoid reflected power to the amplifier and hence increase the power efficiency. These couplings lead to very different biasings of the plasma discharges as it is explained in the Raizer book ${ }^{1}$. In direct coupling, DC current are allowed and the averaged (over one RF period) potential on the electrode is zero while the averaged plasma potential is biased at a fraction of the RF potential depending on the asymmetry of the discharge ${ }^{17}$ : this ratio varies in the range of $1 / \pi$ to 1 . In capacitive coupling, DC currents are not allowed, only displacement currents are, so that the electrode is negatively biased to equal the electron flux to the ion 


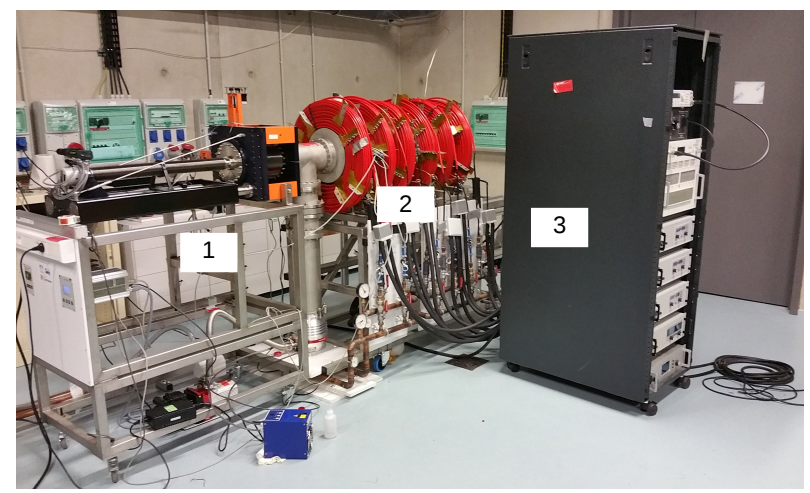

Figure 1. Picture of Aline device. The 3D manipulator (1) in which the probe arm is mounted is connected to the vacuum chamber (2) and the turbo pump. The magnetic red coils are connected to the 3 power dc supplies (bottom of (3)) while the rf electrode is connected to the rf amplifier (in the middle of (3)).

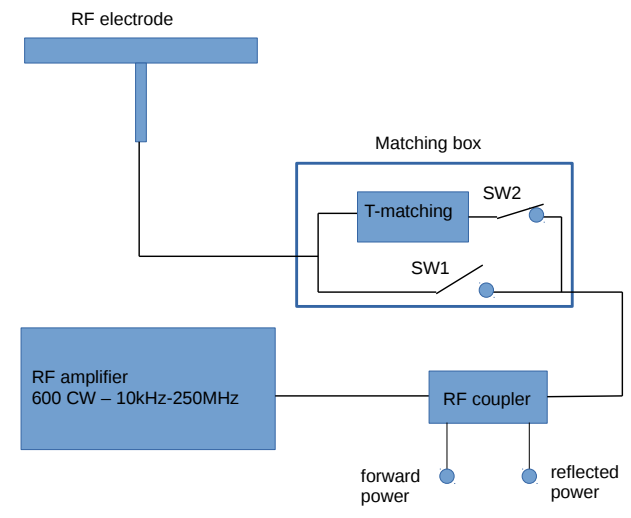

Figure 2. Sketch of the amplifier connected to the RF electrode. Between them the RF coupler is used to measure both forward and reflected power, and the matching box can either be shunt by the switch 1 (Direct coupling) or used as a $\mathrm{T}$ matching with switch 2 (capacitive coupling).

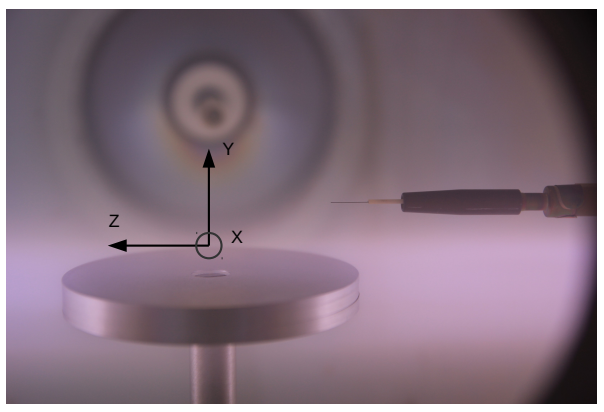

Figure 3. Picture of the probe and the RF electrode. 


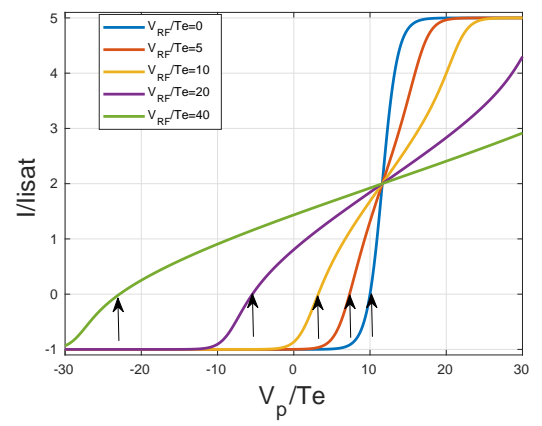

Figure 4. uncompensated I-V characteristics for different RF potentials

one. As this means that almost all the electron are repelled, the negative bias on the electrode is close to the RF potential peak amplitude.

In the present work, the RF power has been scanned from $-16 \mathrm{dBm}$ to $-5 \mathrm{dBm}$ per step of $-1 \mathrm{dBm}$. The RF amplifier increases this signal by $57 \mathrm{dBm}(+/-1 \mathrm{dBm})$ according to the frequency, which leads to a power scan from $12 \mathrm{~W}$ to $160 \mathrm{~W}$. Both types of coupling have been tested and presented in the next sections.

\section{B. The probe}

The main diagnostics available is a RF compensated Langmuir probe from the Hiden Company ${ }^{30}$. This probe is a cylindrical tip of $1 \mathrm{~cm}$ long and $0.15 \mathrm{~mm}$ in diameter as depicted in figure 3 . It is equipped with a compensation electrode and with small inductors able to cut off RF currents at their resonant frequencies which have been chosen according to the working frequencies (between 25 and $40 \mathrm{MHz}$ ). It can be noticed that both the 1st and 2nd harmonic of the RF frequency must be compensated due to potential rectification by the sheath. The RF compensation is essential to measure the real temperature and floating potential, because RF currents can distort the I-V characteristics as shown in figure 4. These I-V characteristics are plotted according to the following equation ${ }^{17}$ :

$$
I_{p}=S_{1} j_{i}\left\langle\frac{\exp \left(\phi_{r f}\right)-\exp \left(\phi_{p}\right)}{\exp \left(\phi_{r f}\right)+S_{1} / S_{2} \exp \left(\phi_{p}\right)}\right\rangle
$$

with $j_{i}$ the ion saturation current density, $\phi_{r f}=10+\frac{V_{r f}}{T_{e}} \sin (\omega t), V_{r f}$ is the rf potential amplitude, $S_{1}$ the probe area, $S_{2}$ the wall area and $\phi_{p}=V_{p} / T_{e}$ the probe potential. $T_{e}$ is here expressed in eV. Usually $S_{2} \gg S_{1}$ and thus the current can be expressed as a simple sheath current while if $S_{1}=S_{2}$ the current becomes a tanh function of the rf potential. The plotted I-V characteristic is averaged over one RF period.

\section{I-V CHARACTERISTICS}

The I-V characteristics have been treated according to 2 different methods. The first is a fit of the ion and exponential part of the characteristics (as explained in the next section) and the second is based upon the OML theory. But the primary condition to make the characteristics usable is the low level of current noise when sweeping the voltage on the probe. The probe voltage is swept from -70 to $+70 \mathrm{~V}$ by $0.02 \mathrm{~V}$ step at an acquisition frequency of $65 \mathrm{KHz}$. Each presented IV characteristic have been averaged 20 times to lower the noise leading to high quality signals able to be derived without too many uncertainties. Several $\mathrm{I}-\mathrm{V}$ characteristics are plotted in figure 5, each of them corresponding to a y position (see figure 3 ) between -5 and $+35 \mathrm{~mm}$ per $1 \mathrm{~mm}$ step. The electrode upper edge is at $\mathrm{y}=-7 \mathrm{~mm}$ for this measurement.

\section{A. Fit of the I-Vs}

The fit of the ion part and exponential part is a fast method to deduce the ion density $n_{i}$, the electron density $n_{e}$ and the electron temperature $T_{e}$ in a single operation. The fit is achieved thanks to a non linear 


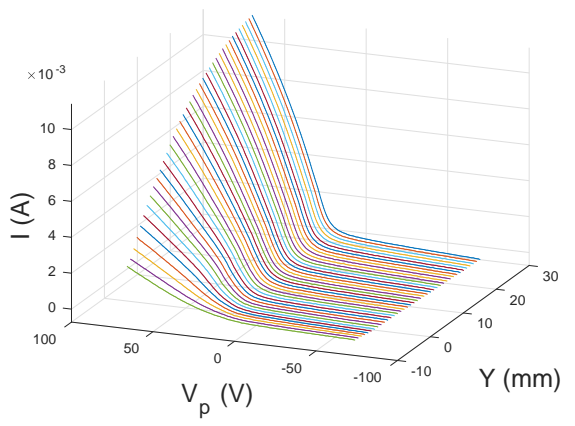

Figure 5. Averaged I-V characteristics for different positions along y axis.

method provided in Matlab software able to deduce each free parameter by successive iteration using a least square method. The advantage of the method is the robustness while the starting parameters (here $n_{i}, n_{e}$ and $T_{e}$ ) are not too far from the final ones. The fit function (equation 2) takes into account the sheath expansion in the ion part according to a Child Langmuir law to deduce the size of the sheath surrounding the probe and the classical exponential part for Boltzmann electrons collected by the repelling probe. The probe potential $V_{p}$ is thus always lower than the plasma potential $V_{p l}$. The last one is deduced from the maximum of the first derivative of the I-V characteristic with a good accuracy (less than $1 \mathrm{~V}$ ) according to the quality of the IVs which have even been smoothed in a second pass.

$$
\begin{array}{r}
I\left(V_{p}\right)=-I_{i}-\alpha\left(V_{p l}-V_{p}\right)^{\frac{3}{4}}+ \\
I_{e} \exp \left(-\frac{V_{p l}-V_{p}}{T_{e}}\right) \\
n_{e}=I_{e} \cdot e^{-1.5} S p^{-1} \sqrt{2 \pi m_{e} / T e} \\
n_{i}=\frac{I_{i}}{e C_{s} S_{p}}
\end{array}
$$

$I_{i}$ and $I_{e}$ are the ion and electron saturation currents respectively, $S_{p}$ is the probe area, $m_{e}$ the electron mass, $C_{s}$ the ion acoustic speed and $\alpha$ is a coefficient depending on the sheath expansion around the probe when collecting ions. To check the reliability of the method, one can compare $n_{i}$ and $n_{e}$ which should theoretically be equal. In most of I-V studies, both of them can be very different, a factor 2 or more is often observed. The advantage of this method is that the $\alpha$ coefficient is just a free parameter to fit perfectly the characteristics but it is not used to deduce any physical parameter, which is suitable according to big uncertainties involved in the sheath expansion.

\section{B. OML theory applied to the I-V char.}

The classical Orbital Motion Limit (OML) theory has been applied to the ion part using an $I^{2}$ method while the temperature has been deduced from the derivative of the log of the electron current, which means that ion current must first be deduced from a previous temperature evaluation. Next iterations are performed to converge to the most probable temperature and density. This method has been successfully used in other rf plasma with the same cylindrical probe ${ }^{33}$. In both methods, the floating potential is always at $\mathrm{I}=0$ and the plasma potential at the maximum of the first derivative. This OML method is widely documented in Langmuir and Bohm works ${ }^{24,34}$. The advantage of the OML theory is to deduce the ion density from the ion part even when the probe is located inside the sheath while the fit method applied to the exponential part does not work anymore due to distortion of the I-V characteristic inside the sheath. Of course, the density deduced from OML theory inside the sheath is not the good one because the ion EDF (Energy Distribution Function) is not Maxwellian, but this theory remains good in the plasma up to the sheath edge, and by continuity a sharp transition in the density can be seen over the sheath edge. In the sheath, ions are accelerated so that the mean velocity is higher. At constant flux, the ion density should then be lower than the one deduced from the OML theory. But again, the interest is to obtain a continuous density profile to find out the sheath edge, and this can only been done using ion flux. 
Table I. scan parameters.

\begin{tabular}{|c|c|c|}
\hline coupling & direct & capacitive \\
\hline rf power scan $(\mathrm{dBm})$ & $41: 1: 52$ & $41: 1: 52$ \\
\hline y position scan $(\mathrm{mm})$ & $1: 1: 46$ & $1: 1: 46$ \\
\hline y electrode position $(\mathrm{mm})$ & 0 & 0 \\
\hline gas type & $\mathrm{He}$ & $\mathrm{He}$ \\
\hline gas pressure $(\mathrm{Pa})$ & 3.4 & 3.4 \\
\hline
\end{tabular}

\section{MEASUREMENTS INSIDE AND OUTSIDE THE RF SHEATH}

\section{A. Experimental protocol}

This section is dedicated to the analysis of plasma parameters resulting from I-V characteristic processing with both methods. The main goal of these experiments is to measure the potential profile from few centimeters off the $\mathrm{rf}$ electrode (outside the $\mathrm{rf}$ sheath) to very small distance (as low as $1 \mathrm{~mm}$ ) to its surface which is now located at $\mathrm{y}=0 \mathrm{~mm}$. According to the typical size of the $\mathrm{rf}$ sheath $(1 \mathrm{~cm})$, one can even expect a potential profile inside the sheath (1 mm space step). To do so, a position scan has been performed to reconstruct the density profile for each step of the a power scan mentioned earlier. This has been done for both $\mathrm{rf}$ couplings (capacitive and direct). For each of the 46 positions composing a profile, an I-V characteristic has been recorded. In total, $1104 \mathrm{I}-\mathrm{V}$ characteristics have been measured thanks to our automated measurement software controlling the manipulator, the probe and the RF power at the same time. All parameters are reminded in table $\mathrm{I}$.

\section{B. Potential profiles within the sheath}

By plotting the measured floating potential $V_{f l}$ (figures 6 and 9) and plasma potential $V_{p l}$ (figure 7 and 10) profiles along the $y$ axis, one can measure the sheath thickness and the plasma potential as a function of the rf power in both couplings. The floating potential profiles are easy to determine from the I-V characteristics (at $\mathrm{I}=0$ ) and exhibit a quasi constant slope in the presheath (at least $2 \mathrm{~cm}$ away from the electrode) and a sudden drop at the sheath edge. The plasma potential profile can be plotted from the maximum of the first derivative of the I-V characteristic outside the sheath. Within the sheath it tends to strongly increase due to the distortion of the velocity distribution function. This property could also be used to detect the sheath edge.

The classical definition of the floating and plasma potential are used outside the sheath: the floating potential is the potential of a floating collecting surface at $\mathrm{I}=0$ and the plasma potential is the potential seen by a transparent probe collecting no charge. Within the sheath, both definitions are the same except that the EDF is not a Maxwellian anymore because electrons are repelled while ions are accelerated. The potential drop $\Delta \phi_{f l}$ between the plasma potential and the floating potential should be maximum at the sheath edge $\left(j_{e}(y=s)=j_{e}\right)$ and $0\left(j_{e}(y=0)=j_{i}\right)$ at the rf electrode surface in capacitive coupling according to equation 5. In direct coupling there is a dc current $\Delta j$ flowing between the rf electrode and the wall. This one is mostly electronic for a rf electrode much smaller than the grounded wall, and thus the floating potential should be lower than expected. At low current $\Delta j \ll j_{e}$, the difference should not be visible.

$$
\Delta \phi_{f l}(y)=\ln \left(\frac{j_{i}}{j_{e}(y)}\right)
$$

\section{Direct rf coupling}

In direct coupling (fig. 6 and fig. 7), both floating and plasma potentials increase with the applied rf potential because the averaged potential on the electrode remains at $0 \mathrm{~V}$ while the plasma is biased at a fraction of the rf potential amplitude. One can see that the floating potential does not converge to $0 \mathrm{~V}$ due to electrostatic perturbations of the probe within the sheath ${ }^{18}$.

For a symmetric rf discharge, i.e. $S_{1}=S_{2}$, with $S_{1}$ the rf electrode area and $S_{2}$ the grounded wall area, and with small rf sheath capacitance, the time averaged plasma potential is $V_{r e c}=\Delta V_{f l}+V_{r f} / \pi^{35}$ with 
$\Delta V_{f l}=\Delta \phi_{f l} / T_{e}$ (see equation 7). For a high capacitive sheath, the averaged plasma potential tends to $V_{s a t}=\Delta V_{f l}+V_{r f}$. More sophisticated models have been derived at relatively low frequency ${ }^{16}$ and using Bessel functions first applied to Langmuir probes ${ }^{36}$ and then to rf discharges $5,13,37,38$. Measurements of the average plasma potential have also been compared to the models ${ }^{13}$ in an Argon plasma. Between these two regimes, a general formula is given by eq. (18) ${ }^{\text {b }}$ but the model the most able to fit the measured potential here is the saturated regime given by equation (30) in the last reference. According to this model the time average plasma potential is :

$$
\begin{gathered}
\langle\phi\rangle=\Delta \phi_{f l}+\ln \left(\frac{S_{1} I_{0}\left(\gamma_{2} \phi_{r f}\right)+S_{2} I_{0}\left(-\gamma_{1} \phi_{r f}\right)}{S_{1}+S_{2}}\right) \\
\Delta \phi_{f l}=-\ln \left(2 \pi \frac{m_{e}}{m_{i}}\right)
\end{gathered}
$$

where $\gamma_{1}=\frac{C_{1}}{C_{1}+C_{2}}$ and $\gamma_{2}=\frac{C_{2}}{C_{1}+C_{2}}$ with $C_{1}$ and $C_{2}$, the rf sheath capacitance at the electrode and at the wall. It has been shown ${ }^{17}$ that this model can also be used for a magnetized flux tube connected to a rf electrode. In that case the capacitance $C_{2}$ is replaced by $C_{\perp}+C_{2}$ with $C_{\perp}$ the equivalent transverse capacitance of the flux tube exchanging rf currents. It means that both a magnetized flux tube and an asymmetric discharge exhibit the same behavior in the case of a direct coupling.

Applying these equations to experimental measurements, one can see in figure 8 that the plasma and floating potentials measured by the probe as a function of rf potential increase almost linearly up to $70 \mathrm{~V}$ and then saturate. The linear part of the measured plasma potential curve must be higher than $V_{\text {rec }}$ and lower than $V_{\text {sat }}$ which are the lowest and highest theoretical values. But it can also be well fitted by equation 6 with $S_{1}$ the real electrode area, $S_{2}=3 S_{1}$, which is far from the real wall area (true $S_{2}=100 S_{1}$ ), $C_{1}=\varepsilon_{0} S_{1} / d_{1}$ and $C_{2}=\varepsilon_{0} S_{2} / d_{2}$ with $d_{1}=5 \mathrm{~mm}$ and $d_{2}=50 \mathrm{~mm}$. $C_{1}$ is close to the true sheath capacitance deduced from he sheath thickness while $C_{2}$ has been adjusted to fit the plasma potential curve. The conclusion of the fit is that the real plasma discharge is far much complex than the model in which density and plasma potential outside the sheath are considered constant. The rf ionization occurs close to the rf electrode, outside the sheath, because electrons are stochastically heated by the rf sheath ${ }^{39}$. This local heating can not be seen in the direct coupling profile but will be in the capacitive coupling profiles.

Finally, one can check that the gap between plasma and floating potential remains constant at all rf potential values. This gap should be equal to equation 7 yielding $17.7 \mathrm{~V}$ for $T_{e}=5 \mathrm{eV}$. Actually the measured value is $10.8 \mathrm{~V}$, i.e. $7 \mathrm{~V}$ lower than expected. This discrepancy can be explained either by a wrong temperature $T_{e}$ or from the different collecting areas for each species. The deviation in temperature can be due to a non-Maxwellian EEDF making an accurate evaluation of the temperature very difficult. But assuming a good temperature, for such a cylindrical probe, the gap can be adjusted from the effective collecting areas for electrons $S_{e}$ an ions $S_{i}$ according to equation 8.

$$
\Delta \phi_{f l 2}=-\ln \left(2 \pi \frac{m_{e}}{m_{i}}\right)-\ln \left(\frac{S_{e}}{S_{i}}\right)
$$

Assuming that the ion collecting area is the probe tip surrounded by a $3 \lambda_{D e}$ thick sheath $^{7}$ and the electron collecting area is the actual tip area, the $7 \mathrm{~V}$ drop can be explained. We have taken in this calculation the averaged temperature and densities plotted in next section : $T_{e}=5 \mathrm{eV}$ and $n=2.510^{16} \mathrm{~m}^{-3}$.

The last discussion about the direct coupling potential concerns the saturation of the plasma potential at 70-75 V while the rf potential still increases. To explain that, one assumes that the electron current at the electrode also saturates, and it can be calculated by equation $(31)^{17}$. Actually this formula must be corrected because the area $S_{1}+S_{2}$ should appear at the numerator as follows :

$$
\left|I_{1}\right|=j_{i} S_{2}\left(1-\frac{S_{1}+S_{2}}{S_{1} I_{0}\left(\phi_{r f}\right)+S_{2}}\right)
$$

where $I_{1}$ the current collected by the electrode in the high sheath capacitance regime and $I_{0}$ the modified Bessel function of the first kind. The rf potential limit for the saturation can then be deduced from $\left|I_{1}\left(\phi_{r f}\right)\right|>$ $I_{\text {esat }}=j_{e} S_{1}$. Beyond this limit the electron flux must be accelerated by the sheath in which the potential drop 


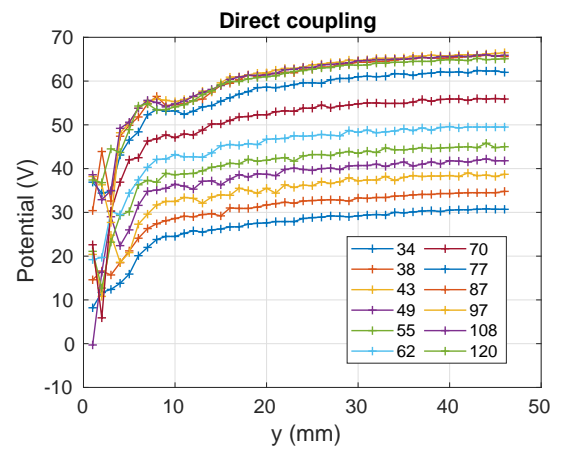

Figure 6. Floating potential measured by the probe with respect to the position along y axis in direct coupling for $12 \mathrm{rf}$ potential amplitudes (legend in volts).

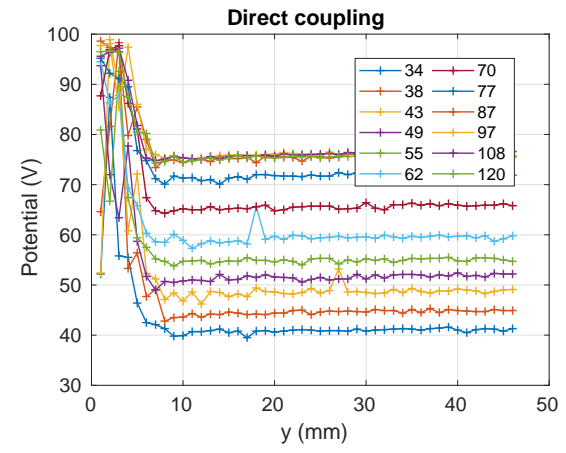

Figure 7. Plasma potential measured by the probe with respect to the position along y axis in direct coupling for $12 \mathrm{rf}$ potential amplitudes (legend in volts).

is reversed. Increasing the rf potential increases the sheath voltage as well, but not the plasma potential, which explains the saturation of the plasma potential ${ }^{40}$. From equation 9 , with $S_{1}$ and $S_{2}$ the effective electrode and wall areas, the limit rf potential is equal to $29 \mathrm{~V}$, instead of $80 \mathrm{~V}$ in figure 8 . This means that the real wall area or the plasma density close to the wall is overestimated as already seen in the fit parameters of equation 6. This is not a surprise as the density decreases along the profile. Moreover formula 9 is only valid for $\left|I_{1}\right|<I_{\text {esat }}$. As a consequence, an accurate value of the potential is then not possible to get. Such electron sheaths have already been studied and measured ${ }^{18,41,42}$, where it is shown that the modification of the electrode area is a key parameter to reverse the electric fied in the sheath.

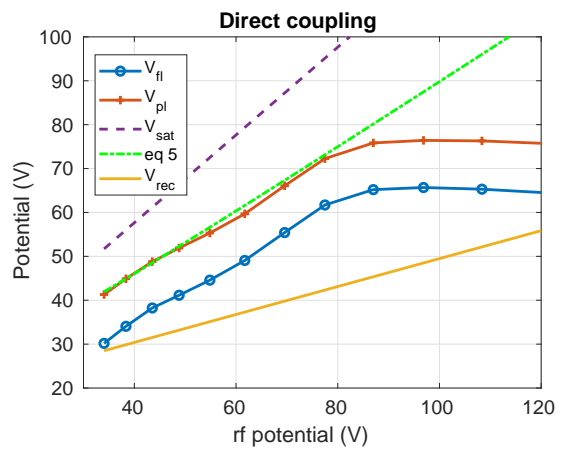

Figure 8. Floating $V_{f l}$ and plasma $V_{p l}$ potentials measured by the probe with respect to the applied rf potential at the electrode in direct coupling. $V_{r e c}=\Delta V_{f l}+V_{r f} / \pi$ and $V_{s a t}=\Delta V_{f l}+V_{r f}$. 


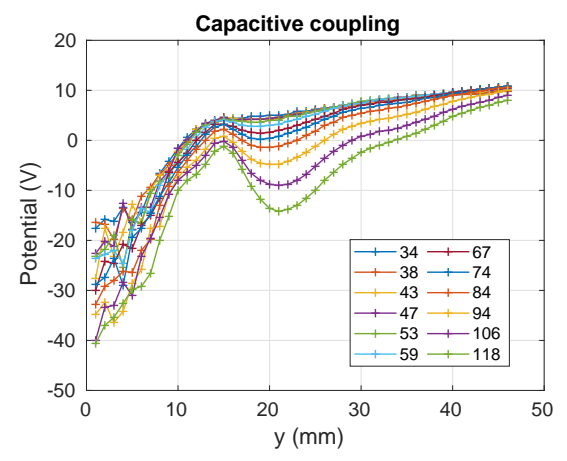

Figure 9. Floating potential $V_{f l}$ measured by the probe with respect to the position along y axis in capacitive coupling for $12 \mathrm{rf}$ potential amplitudes (legend in volts).

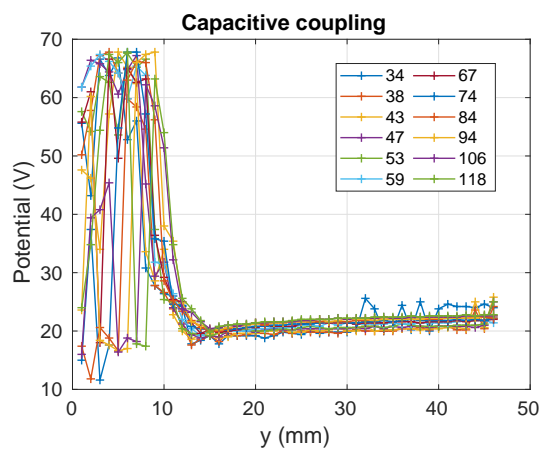

Figure 10. Plasma potential measured by the probe with respect to the position along y axis in capacitive coupling for $12 \mathrm{rf}$ potential amplitudes (legend in volts).

\section{Capacitive coupling}

In capacitive coupling, all the rf potential grows inside the rf sheath due to the negative self biasing of the rf electrode to repel electrons. This capacitive coupling is ensured by a matching box (T matching) composed of two capacitors and one tunable inductor. The floating potential profiles are almost identical outside the rf sheath. Inside the sheath, the potential gap increases with the rf potential (fig. 9). The floating potential value close to the rf electrode should be almost equal to the electrode potential as this potential depends on the electron flux arriving onto the probe. Deeper into the sheath, the electron flux decreases leading to a decrease of the floating potential. Here the lowest floating potential that can be measured by the probe depends on the first value of the probe potential scan which is $-70 \mathrm{~V}$. The floating potential outside the sheath is close to the value given by equation 8 . Nevertheless one can see that the higher the rf potential, the deeper is the potential well in front of the sheath edge. This well is probably due to electron heating as explained above. This effect is more visible in the present case as the total potential drop in the sheath is much higher than the rf potential because of the matching box. As a matter of fact the self biased electrode potential $V_{\text {bias }}$ can reach -500 volts while the rf potential measured before the matching box is a slightly higher than $100 \mathrm{~V}$ as shown in figure 11.

As for the plasma potential profiles (fig. 10) it is clearly flat outside the sheath while it increases strongly at the sheath edge. The only way here to retrieve the plasma potential within the sheath is to use an emissive probe $^{18}$. Then taking the averaged potentials over the last 5 points of the profiles (the farthest from the electrode), one can plot both plasma and floating potential as a function of the rf potential (see fig. 12).

And last point, the potential gap between plasma and floating potentials is roughly $13 \mathrm{~V}$ which is in agreement with equation 8 with $T_{e}=6 \mathrm{eV}$. This gap is constant in the first part of the curve below $80 \mathrm{~V}$ for the rf potential. Beyond this value, the potential well extends up to few centimeters away from the sheath edge and decreases the floating potential due to the increase of $T_{e}$ in this "heating" region. The typical size of the potential well is of the order of the mean free path for electron-neutral collisions $(\approx 2 \mathrm{~cm})$. 


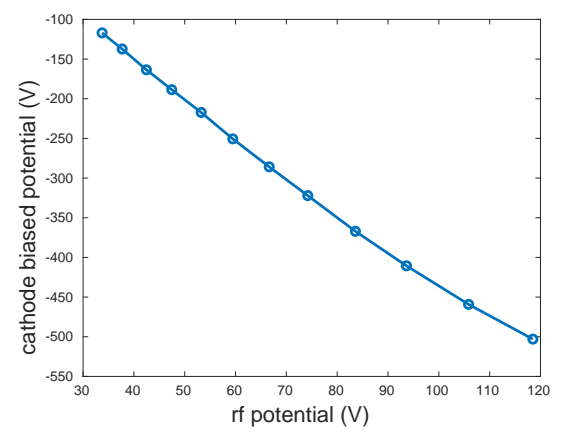

Figure 11. Amplitude of the dc biased potential at the electrode $V_{\text {bias }}$ with respect to the rf potential at the entrance of the matching box (capacitive coupling).

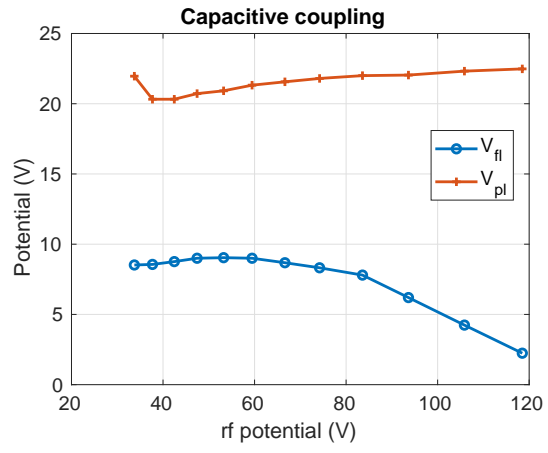

Figure 12. Floating $V_{f l}$ and plasma $V_{p l}$ potentials measured by the probe with respect to the applied rf potential at the electrode in capacitive coupling

\section{Densities and temperatures}

For direct coupling and from the fit method, ion and electron densities are plotted in figure 13 and electron temperature in figure 15. The first remarkable result is the quasi square root dependence of the density vs the RF power (power 0.6 for $n_{e}$ and 0.63 for $n_{i}$ ). The second result is the relative small discrepancy between $n_{i}$ and $n_{e}$ with the fit method which takes into account the expansion of the sheath. The average $n_{i} / n_{e}$ ratio is equal to 1.34. The temperature is relatively constant over the whole range of power as expected and is roughly equal to $4.2 \mathrm{eV}$. We have a pretty good confidence in the quality of the I-V characteristic fits as the Mean Squared Error is indeed lower than $10^{-10}$ and level of noise in the temperature curve fairly low. Here $n_{e}$ seems much more reliable than $n_{i}$ for the fit method because it depends on the exponential part of the curve for which currents are much higher compared to ion currents.

In capacitive coupling the density plot showed in figure 14 exhibits almost the same values as in direct

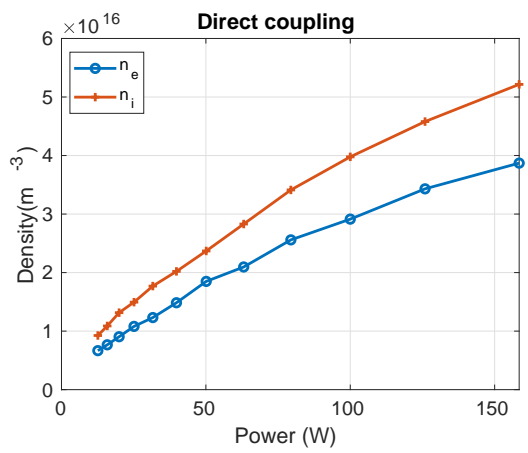

Figure 13. Ion and electron densities from the fit method with respect to rf power in direct coupling. 


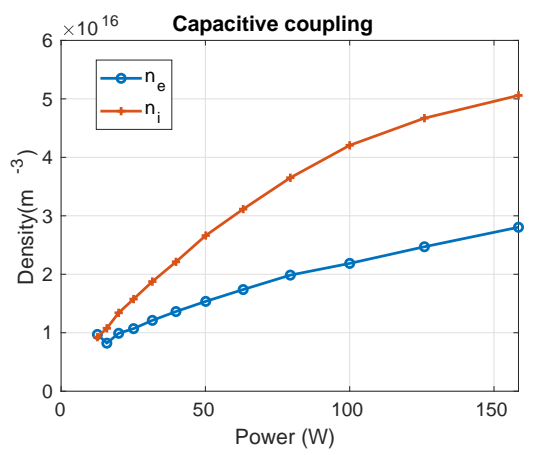

Figure 14. Ion and electron densities from the fit method with respect to rf power in capacitive coupling.

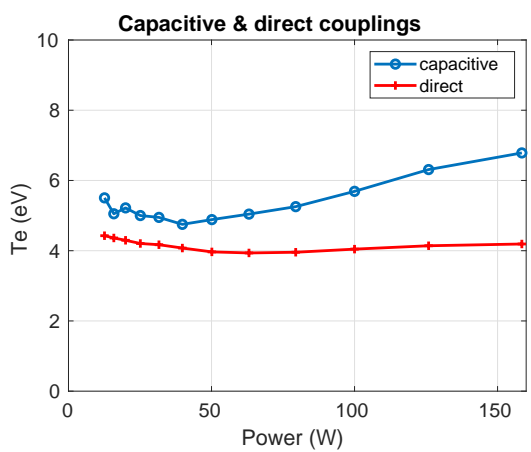

Figure 15. Electron temperature with respect to rf power in both capacitive and direct couplings

coupling for $n_{i}$ while $n_{e}$ is two times lower. The temperature is constant at low level of rf power and increases at higher level due to near heating effect of the electrode. The probe is not far enough (several mean free path) to sense a pure thermal distribution of electrons, which explains the temperature rise from $5 \mathrm{eV}$ to $7 \mathrm{eV}$ at the highest rf power in figure 15 .

\section{RF SHEATH THICKNESS}

As explained in the introduction, measurements of the rf sheath thickness are not widespread in the plasma community. A wide panel of the potential diagnostics are presented in this reference ${ }^{18}$ and the conclusion is that sheaths, and especially rf sheaths, are pretty complicated to diagnose and to understand.

The present technique is based on the rf compensated cylindrical Langmuir probe, thin enough $(0.15 \mathrm{~mm}$ in diameter) to not disturb too much the potential distribution within the rf sheath. Even if the probe disturbs the equipotential lines within the sheath ${ }^{18}$, it does not ruins its ability to detect the sheath edge, which means that the floating potential measured is not the sheath potential but vary with the same trend than the sheath potential. That is why Langmuir probe may be a good candidate to determine the sheath thickness.

The averaged sheath thickness can be deduced either from the potential profiles showed in figures 6 and 9 or from the density profiles given by the OML method inside the sheath. These density profiles are plotted in figure 16 and 17. The most astonishing thing is the accuracy of the density profiles inside the sheath. The sheath edge is then much easier to determine than in the potential profiles even if the plasma density is overestimated compared to the fit method ( 3 times higher). At least the results from both methods can be compared.

\section{A. Direct coupling}

In direct coupling the sheath thickness does not change much with the rf power. This is due to the increase of the density which decreases the Debye length with the rf power, which balances the total sheath expansion with the rf potential. Taking the sheath edge at the maximum of the second derivative of the potential profile 


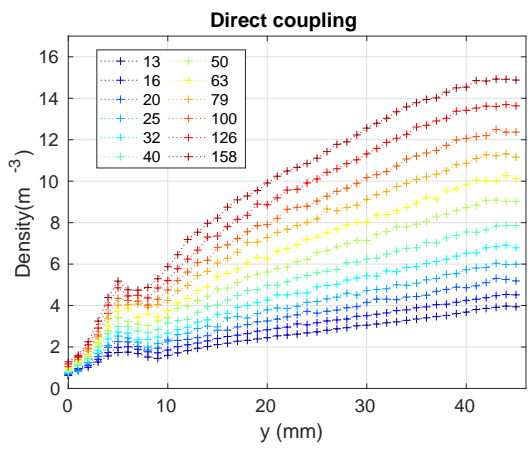

Figure 16. Ion density profiles from OML method with respect to the position along y axis in direct coupling (legend : rf power in Watts).

(Vfl method) yields to a rather constant thickness of $6 \mathrm{~mm}$. But using the ion density profiles derived from the OML method, and taking the position at the first maximum of density in figure 16 the average value for the thickness is $3.3 \mathrm{~mm}$ and remains almost the same for all rf power levels. The third method (Vpl method) consists in measuring the position at half the potential leap near the electrode to detect the sheath edge, as a matter of fact the method to find out the plasma potential does not work anymore inside the sheath and its value tends to increase very quickly. In figure 18, the three measured thicknesses are compared with the Child Langmuir law, Lierberman Law $^{8}$ and a semi analytic model ${ }^{11}$. Other experimental comparison with Godyak and Lieberman models for the electric properties of the sheath are presented in ref $^{44}$ for collisional and asymmetric discharges. The Godiak model ${ }^{10}$ is rather complicated to solve and is not plotted here. But it exhibits a $V_{r f}^{\frac{1}{2}}$ dependence instead of $V_{r f}^{\frac{3}{4}}$. The Wang model is simplified by considering a factor 3 with the CL law for the same range of pressure (20-30 mTorr or 2.7-4 Pa and $\mathrm{P}=3.5 \mathrm{~Pa}$ in our measurements). This model computes the sheath thickness in an Argon plasma considering slightly lower mean free path. The result for the sheath thickness does not depend on the ion mass so that one can expect the same value in a Helium plasma. For the Child Langmuir law, one assumes $\alpha=1$, for Lieberman's law $\alpha=\sqrt{50 / 27}$, and $\alpha=3$ for Wang semi analytic model in the following expression :

$$
\frac{s}{\lambda_{D e}}=\alpha\left(\frac{V_{r f}}{T_{e}}\right)^{\frac{3}{4}}+5
$$

where $V_{r f}$ is used instead of the average potential gap in the sheath because the plasma potential $V_{p}$ saturates at $V_{r f}=80 \mathrm{~V}$ due to the reversal of the rf sheath as seen in figure 8. In the same figure, the average plasma potential is almost equal to the rf potential amplitude $V_{r f}$ while it does not saturate. In equation 10 , the floating sheath thickness is assumed to be equal to $5 \lambda_{D e^{2,7}}$.

The sheath thickness is supposed constant and an averaged value over all $\mathrm{rf}$ power is used to plot $s / \lambda_{D e}$. The temperatures of figure 15 and the electron density $n_{e}$ for direct coupling are used to calculate the Debye length. Figure 18 shows that all measured value overestimates the theoretical sheath thicknesses as CL and Lieberman, but the "n method' measurement is finally very close to the semi analytical model $(\alpha=3)$. Other experimental methods (Vfl and Vpl methods) overestimate again all of the others. The conclusion is that the real average rf sheath thickness in direct coupling is at least 3 times higher than the one given by Child Langmuir law in a low collisional Helium plasma.

\section{B. Capacitive coupling}

In capacitive coupling the sheath thickness is much larger due the matching box which enhances the negative dc biasing at the electrode $V_{\text {bias }}$ as seen in figure 11 . The sheath thickness is no more constant with respect to rf power, and tends to increase. So $s / \lambda_{D e}$ is plotted with the deduced sheath thickness for each $\mathrm{rf}$ power level. Indeed the temperature used to compute the Debye length is now the average of temperatures in figure 15 and the density is taken from figure 14 for the capacitive coupling. Looking at the sheath thickness (see figure 19) as a function of $V_{\text {bias }} / T_{e}$ using density method (n method) and plasma potential method (Vpl method), one can see that both methods lied to very similar results considering the uncertainties (the position 


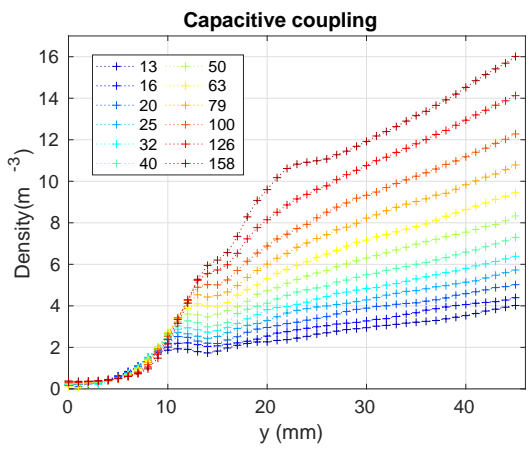

Figure 17. Ion density profiles from OML method with respect to the position along y axis in capacitive coupling (legend : rf power in Watts).

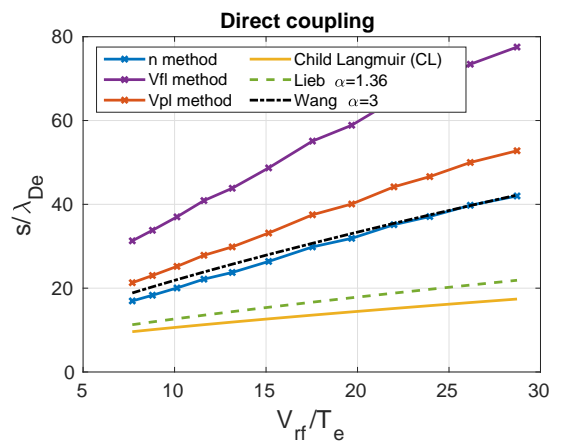

Figure 18. Sheath thicknesses normalized to the Debye length plotted with respect to the rf potential normalized to Te in direct coupling. The 3 measured curves using density method, floating method and plasma potential method are compared to theoretical methods (Child Langmuir, Lieberman method and Wang method.

accuracy is $1 \mathrm{~mm}$, which means at least $10 \%$ of relative error). Again the coefficient $\alpha=3$, also matching the Wang's model, seems to fit well as it was the case in direct coupling. And again CL and Lieberman laws underestimate the sheath width.

Here the floating potential method is not plotted because it is hard to find a relevant criterion for the sheath edge. At a first sight in figure 9 it seems constant if we consider the sheath edge where the first derivative is zero. But if we plot $s / \lambda_{D e}$ with a constant sheath thickness the power law is no more verified.

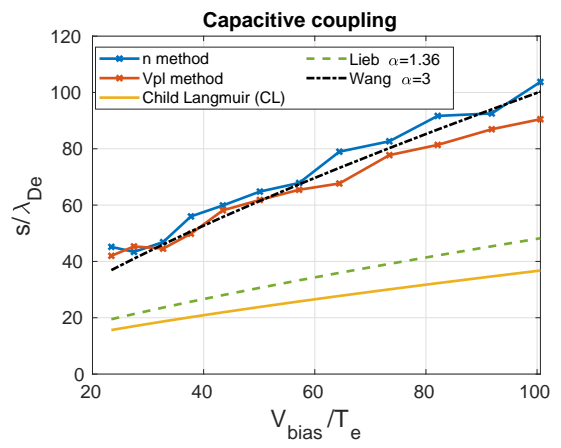

Figure 19. Sheath thicknesses normalized to the Debye length plotted with respect to the rf potential normalized to Te in capacitive coupling. The 2 measured curves using density method and plasma potential method are compared to theoretical methods (Child Langmuir, Lieberman method and Wang method. 


\section{CONCLUSION}

The main goal of this work was to check the ability to make measurements within a rf sheath using a rf compensated and a thin Langmuir probe in a plasma without magnetic field. Measurements have been performed in the Aline plasma device equipped with a rf electrode and a probe mounted on a 3D manipulator to draw potential and density profiles. The potential profiles have been plotted from the rf electrode position up to several centimeters into the core plasma. It has been shown that they are smooth enough to be analysed, even very close to the rf electrode, inside the rf sheath. While the floating potential is easy to deduce from the I-V characteristics, the plasma potential has been extracted from the maximum of the first derivative of the characteristics using smoothing methods. The plasma potential profiles exhibit a small slope due to the presheath in the core plasma while in the sheath it is not reliable due to I-V characteristics distortion. The floating potential profiles reveal a strong decrease of the potential over the sheath edge. All these profiles allow to detect the sheath edge.

The self biasing of the plasma due to the rf power has been checked with respect to several models in direct coupling. We found that the most adapted is a capacitive saturated model taking into account the size of each electrode. Nevertheless this model works only under a potential threshold above which the sheath potential is reversed to allow electron acceleration. This phenomenon has been explained by the fact that the electron saturation current drained by the rf electrode is not high enough to compensate the ion current collected at the grounded wall. The higher the plasma biasing, the higher the positive current at the wall. As a consequence, the total ion current at the wall can be higher than the electron saturation current over the electrode area, hence the need of a "reversed" sheath in order to accelerate electrons on the electrode side. In capacitive coupling, the electrode is negatively biased so that the plasma potential remains low and weakly disturbed by rf oscillations.

The potential drop between the plasma and floating potential profiles remains constant as a function of the rf power. This potential drop has been used to compute the floating sheath thickness around the probe in the low probe voltage range $\left(V_{p}<T_{e}\right)$ considering that ion collecting area is not the probe tip but the sheath edge surrounding this tip. In the present case it yields a typical sheath thickness of 3 Debye lengths.

To compare these measured sheath thicknesses to the Child-Langmuir law at high rf potentials, the main plasma parameters (density and temperature) have been deduced from the analysis of the IV characteristics in the quasi neutral plasma. This has been done using 2 methods, a "fit method" and the classical "OML method" for ions. The "fit method" works well to find out both ion and electron densities with a relative good agreement (less than a factor 2 between them) as well as for the electron temperature. This method does not apply anymore within the sheath due to the non-Maxwellian EEDF. The OML theory based on the ion part of the characteristics is able to yield a continuous ion density profile from the plasma to the electrode. Even if, within the sheath, the thermal velocity is underestimated (because ions are accelerated) , this method is a good way to determine the sheath edge and then its thickness.

From the comparison of the 3 measurement methods ("density", "plasma potential" and "floating potential" methods) to theoretical laws, the conclusion is that the "density" and "plasma potential" methods give the most reliable results while the floating potential method seems to overestimate the sheath thickness. Nevertheless it leads to a typical average rf sheath thickness 3 times higher than the classical Child Langmuir law, more than 2 times higher than the Lieberman correction but in the same range than a semi analytic model given in another study. The sheath thickness law seems to be independent of the coupling for the same rf power levels. The main conclusion is that the average sheath thickness around a rf electrode is 3 times higher than the classical Child-Langmuir law, applying for relatively high dc potentials. This factor has been obtained using the amplitude of the rf potential in place of the dc potential drop in a ChildLangmuir like formula, in the case of a direct coupling. As a matter of fact, the sheath potential drop can not be used because of the saturation of the self biasing of the plasma, due to the sheath potential reversal at high rf power. In capacitive coupling, the real averaged potential drop in the sheath has been used.

\section{ACKNOWLEDGEMENT}

This work has been carried out within the framework of the French Federation for Magnetic Fusion Studies (FR-FCM) and of the Eurofusion consortium, and has received funding from the Euratom research and training programme 2014-2018 and 2019-2020 under grant agreement No 633053. The views and opinions expressed herein do not necessarily reflect those of the European Commission. 


\section{REFERENCES}

${ }^{1}$ Yuri P. Raizer, Mikhail N. Shneider, Nikolai A. Yatsenko, Radio-Frequency Capacitive Discharges, CRC Press, 1st Edition (1995)

${ }^{2}$ Michael A. Lieberman, Alan J. Lichtenberg, Principles of Plasma Discharges and Materials Processing, WILEY, second edition (2005)

${ }^{3}$ P. Chabert and N. Braithwaite, Physics of Radio-Frequency Plasmas, Cambridge: Cambridge University Press (2011)

${ }^{4}$ V. A. Godyak and A. A. Kuzovnikov, Fiz. Plasmy 1, 496 (1975) [Sov. J. Plasma Phys. 1, 276 (1975)]

${ }^{5}$ H. S. Butler and G. S. Kino, Phys. Fluids 6, 1346 (1963)

${ }^{6}$ K -U Riemann, J. Phys. D: Appl. Phys. 24493 (1991)

${ }^{7}$ P Chabert, Plasma Sources Sci. Technol. 23065042 (2014)

${ }^{8}$ M. A. Lieberman, IEEE Trans. Plasma Sci. 16, 638 (1988).

${ }^{9}$ M. A. Lieberman, IEEE Trans. Plasma Sci. 17, 338 (1989)

${ }^{10}$ V A Godyak and N. Sternberg, Physical Review A, 42, 4, 2299 (1990)

${ }^{11}$ Shiang-Bau Wang and Amy E. Wendt, IEEE Trans. Plasma Sci., 27, 5 (1999)

${ }^{12}$ M A Lieberman, J. Appl. Phys., 65, 4186 (1989)

${ }^{13}$ K. Köhler, J. W. Coburn, D. E. Horme, and E. Kay, J. Appl. Phys., 57, 59 (1985)

${ }^{14}$ Y P Raizer and M N Schneider, Plasma Sources Sci. Technol., 1, 102 (1992)

${ }^{15}$ A. Metze, D. W. Ernie, and H. J. Oskam, J. Appl. Phys. 60, 3081 (1986)

${ }^{16}$ A. M. Pointu, Journal of Applied Physics 60, 4113 (1986)

${ }^{17}$ E. Faudot, Phys. Plasmas 22, 083506 (2015)

${ }^{18}$ Noah Hershkowitz, Physics of Plasmas 12, 055502 (2005)

${ }^{19}$ L. Oksuz and N. Hershkowitz, Phys. Rev. Lett. 89, 145001 (2002)

${ }^{20}$ M-H. Cho, N. Hershkowitz, and T. Intrator, J. Vac. Sci. Technol. A 6, 2978 (1988)

${ }^{21}$ S. Kajita, S. Kado, A. Okamoto, T. Shikama, Y. Iida, D. Yamasaki and S. Tanaka, arXiv:physics/0410187, 12th International Congress on Plasma Physics, 25-29 October 2004, Nice (France)

${ }^{22}$ A. H. Sato, and M. A. Lieberman, J. Appl. Phys. 68 (12), 1990

${ }^{23}$ Hyung-Sik Han, Hyo-Chang Lee, Se-Jin Oh, and Chin-Wook Chung, Physics of Plasmas 21, 023512 (2014)

${ }^{24} \mathrm{H}$ M Mott-Smith and I. Langmuir, Phys. Rev. 28, 727 (1926)

${ }^{25}$ J.E. Allen, R.L.F. Boyd, and P. Reynolds, Proc. Phys. Soc. (London) B70, 297 (1957)

${ }^{26}$ F.F. Chen, J. Nucl. Energy, Pt. C 7, 47 (1965)

${ }^{27}$ I.B. Bernstein, and I.N. Rabinowitz, Phys. Fluids 2, 112 (1959)

${ }^{28}$ Laframboise, J.G. 1966 Univ. Toronto Inst. Aerospace Studies Rept. 100 (June, 1966)

${ }^{29}$ Francis F. Chen, John D. Evans, and Donald Arnush, Physics of Plasmas, 9, 4 (2002)

${ }^{30} \mathrm{P}$ A Chatterton, J A Reese, W L Wu and K Al Assadi, Vacuum, 42, 7, 489-493, (1991)

${ }^{31}$ F F Chen, Plasma Sources Sci. Technol. 21, 055013 (2012)

${ }^{32}$ E. Faudot, S. Devaux, J. Moritz, S. Heuraux, P. Molina Cabrera and F. Brochard, Review Of Scientific Instruments 86, 063502 (2015)

${ }^{33}$ F F Chen, Plasma Sources Sci. Technol. 18, 035012 (2009)

${ }^{34}$ D. Bohm, The Characteristics of Electrical Discharges in Magnetic Fields, A. Guthrie, R.K. Wakerling, Eds., McGraw-Hill, New York 1949 p.77.

${ }^{35}$ V. A. Godyak, Fiz. Plazmy 2, 141 (1976)

${ }^{36}$ A Garscadden and K G Emeleus, Proc. Phys. Soc., 79, 535 (1962)

${ }^{37}$ J H Keller and W B Pennebaker, IBM J Res Dev, 23, 3 (1979)

${ }^{38}$ T D Mantei, J Electrochem. Soc., 130, 1958 (1983)

${ }^{39}$ B P Wood, M A Lieberman and A J Lichtenberg, IEEE Transactions on Plasma Science, 23, NO 1, 89 (1995)

${ }^{40}$ A. Aanesland, C. Charles, R. W. Boswell and M. A. Lieberman, Physics Of Plasmas 12, 103505 (2005)

${ }^{41}$ C. Forest and N. Hershkowitz, J. Appl. Phys. 60, 1295 (1986)

${ }^{42}$ M-H. Cho, N. Hershkowitz, and T. Intrator, J. Appl. Phys. 67, 3254 (1990)

${ }^{43}$ V A Godyak, O A Popov and A H Hanna, Proc. XIII Int. Conf. on Phenom. in Ionized Gases, Berlin, 347 (1977)

${ }^{44}$ M. A. Sobolewski, Appl. Phys. Lett. 70 (8), (1997)

${ }^{45}$ S. Devaux, E. Faudot, J. Moritz, S. Heuraux, Nuclear Materials and Energy, 12, 908 (2017) 